\title{
Attraction-driven aggregation of dipolar particles in an external magnetic field
}

\author{
Gergő Pál, ${ }^{1}$ Ferenc Kun, ${ }^{1,2}$ Imre Varga, ${ }^{1}$ Dóra Sohler, ${ }^{2}$ and Gang Sun ${ }^{3}$ \\ ${ }^{1}$ Department of Theoretical Physics, University of Debrecen, P.O. Box 5, H-4010 Debrecen, Hungary \\ ${ }^{2}$ Institute for Nuclear Research (ATOMKI), Hungarian Academy of Sciences, P.O. Box 51, H-4026 Debrecen, Hungary \\ ${ }^{3}$ Beijing National Laboratory for Condensed Matter Physics and Key Laboratory of Soft Matter Physics, Institute of Physics, \\ Chinese Academy of Sciences, Beijing 100190, China \\ (Received 16 November 2010; published 16 June 2011)
}

\begin{abstract}
We present a detailed experimental study of the aggregation of particles of permanent magnetic moment in an external magnetic field. The experiments are performed with millimeter-sized particles floating on the surface of water. Due to the large size of the particles, thermal noise does not have any relevance in the system; the particles undergo deterministic motion. Experiments are carried out varying the concentration which also controls the relative importance of the dipole-external field and the dipole-dipole interactions. We determined the exponents characterizing the aggregation process and found that the attraction driven aggregation of dipolar particles obeys the Vicsek-Family dynamic scaling. The exponents are found to have a concentration dependence which can be attributed to the change of mobility of clusters and their interaction at higher concentrations.
\end{abstract}

DOI: 10.1103/PhysRevE.83.061504

PACS number(s): 83.10.Tv, 89.75.Da, 87.15.nr

\section{INTRODUCTION}

Structure formation of magnetic particle systems due to self-assembly plays a crucial role in a wide range of phenomena from dipolar colloidal fluids to magnetized granular materials [1]. In a monolayer of dipolar particles the long-range anisotropic interaction and the inherent frustration of the system give rise to very interesting phenomena where the particles self-assemble into various types of structures [2-4]. The presence of such aggregates has a strong effect on the statistical thermodynamic [3,5,6], optical [7], and rheological properties [8,9] of these systems, which calls for a detailed understanding of the process of aggregation.

Depending on the size scale of particles several experimental techniques have been used to investigate the kinetic, dynamic, and statistical thermodynamic features of dipolar particle systems: Cryogenic transmission electron microscopy was used to investigate colloidal dispersions of single domain magnetites $[4,10]$. In the micrometer range monolayers of dipolar particles have been realized by confining particles or holes on the surface of a liquid, in the interface of two fluid layers [11-13], or to the bottom of a fluid container [14,15]. Experiments revealed that the particle system undergoes a cluster-cluster aggregation process which results in chains or more complex branching structures depending on the particle concentration and on the presence or absence of an external magnetic field $[16,17]$. Using an external magnetic field with appropriate orientation various types of crystal structures can also be achieved in magnetic dipolar monolayers [12]. At higher length scales, centimeter sized magnetic particles provide interesting granular assemblies, where, using vibrofluidization techniques, aggregation phenomena and the phase structure of granular fluids-gases can be investigated [18-21]. Using centimeter sized steel balls of permanent magnetic moments the effect of long range anisotropic forces on the angle of repose and avalanche dynamics of granular materials have been studied [22]. The large particle size in these experimental studies offers several advantages; however, it implies some additional difficulties to overcome the frictional barrier. In these cases usually vibrating tables are used to mobilize the particles tuning the frequency and amplitude of the excitations [14,18-21].

In the present paper we investigate the aggregation of macroscopic particles of permanent magnetic dipole moment confined in a plane. In order to decrease the frictional barrier, the particles float on the surface of water in such a way that their dipole moment always falls in the plane of motion. This technique ensures that the translational and rotational motion of the particles is only affected by the Stokes drag force and torque exerted by the liquid [23]. The main advantage of the experimental setup is that using direct optical observations all single particles can be tracked and even the orientation of their dipole moment can be determined any time during the time evolution. Due to the large size of the particles the thermal motion does not play any role in the system. Varying the concentration in a broad range we investigate the dynamics of the aggregation process. Experiments revealed that the attraction driven aggregation of dipolar particles obeys the Vicsek-Family dynamic scaling similarly to diffusion dominated aggregation of dipoles $[11,16]$. The dynamic exponents have a concentration dependence, which can be attributed to the change of the mobility of clusters with their size and to the change of the interaction of clusters as the concentration increases.

\section{EXPERIMENTAL SETUP}

In the experiments millimeter sized metal cylinders magnetized along their axis are attached to swimmers. The cylinders are $\mathrm{NdFeB}$ magnets provided by BorsMagnet [24] having a remanence of $1.1 \mathrm{~T}$. The diameter and height of the metal cylinder are $d_{m}=2 \mathrm{~mm}$ and $h_{m}=10 \mathrm{~mm}$, respectively. The swimmers are cork disks of diameter $d_{s}=25 \mathrm{~mm}$ and height $h_{s}=5 \mathrm{~mm}$ which is sufficient to stabilize the particle on the water surface. The composite particles can swim on the top of water which facilitates the translational and rotational motion. A detailed experimental investigation of the motion of composite particles on the surface of water was carried out in Ref. [23]. It was shown that the motion can be described by taking into account the interaction of pointlike dipoles and by 


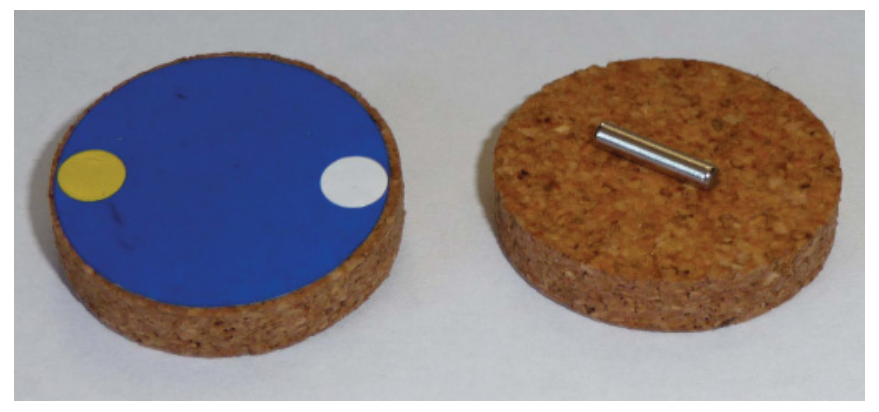

FIG. 1. (Color online) Composite particles used in the experiments. Metal cylinders magnetized along their axis are attached to cork disks which can then float on the surface of water. The top of the disk is colored blue on which the white and yellow spots indicate the orientation of the vector of the dipole moment.

the Stokes drag force exerted by the fluid. The typical magnetic force between two particles was found to be more than an order of magnitude larger than the drag force [23]. In order to facilitate the image processing and particle tracking, the top of the particles is colored to blue on which white and yellow spots indicate the orientation of the dipole vector. Figure 1 presents the construction of the composite particles and the coloring. The experiments are carried out in a square-shaped water container of size $L=100 \mathrm{~cm}$ and height $5 \mathrm{~cm}$. In order to fully control the initial state of the particle system, a hard plastic net with a wooden frame is put into the container which floats on the water surface. The initial configuration of the system is prepared in such a way that the particles are placed on the top of the net with random position and random direction of the dipole moments. The particles start to move at the same time when the net is immersed into the water letting the particles float. We have used a former version of this experimental technique to investigate the pattern formation in binary colloidal monolayers $[23,25]$.

The floating particles undergo translational and rotational motion due to the anisotropic dipole-dipole interaction and due to the action of the homogeneous external magnetic field of Earth. At low concentrations, as soon as the particles become free to move, they rotate and get aligned with the Earth magnetic field. Increasing the concentration, however, the local dipole field of the interacting particles can overcome the external field strength, resulting in an interesting change in the dynamics and structure of the system. The particle concentration $\phi$ is defined as the coverage, that is,

$$
\phi=N R^{2} \pi / L^{2},
$$

where $R=d_{s} / 2$ denotes the radius of cork disks and $N$ is the total number of composite particles. The investigation of the dynamics of the aggregation process was carried out in the range $\phi \leqslant 0.16$, where $\phi$ was controlled by varying the number of particles $N=50,100,150,200,250,300$. At each concentration measurements were repeated 30 times recording them by a digital video camera with a frame rate 15 frames/s. The relatively large particle size and the high resolution of the camera let us fully automatize the image analysis. All single particles are tracked frame by frame determining also the vector of the dipole moment. We note that above the upper
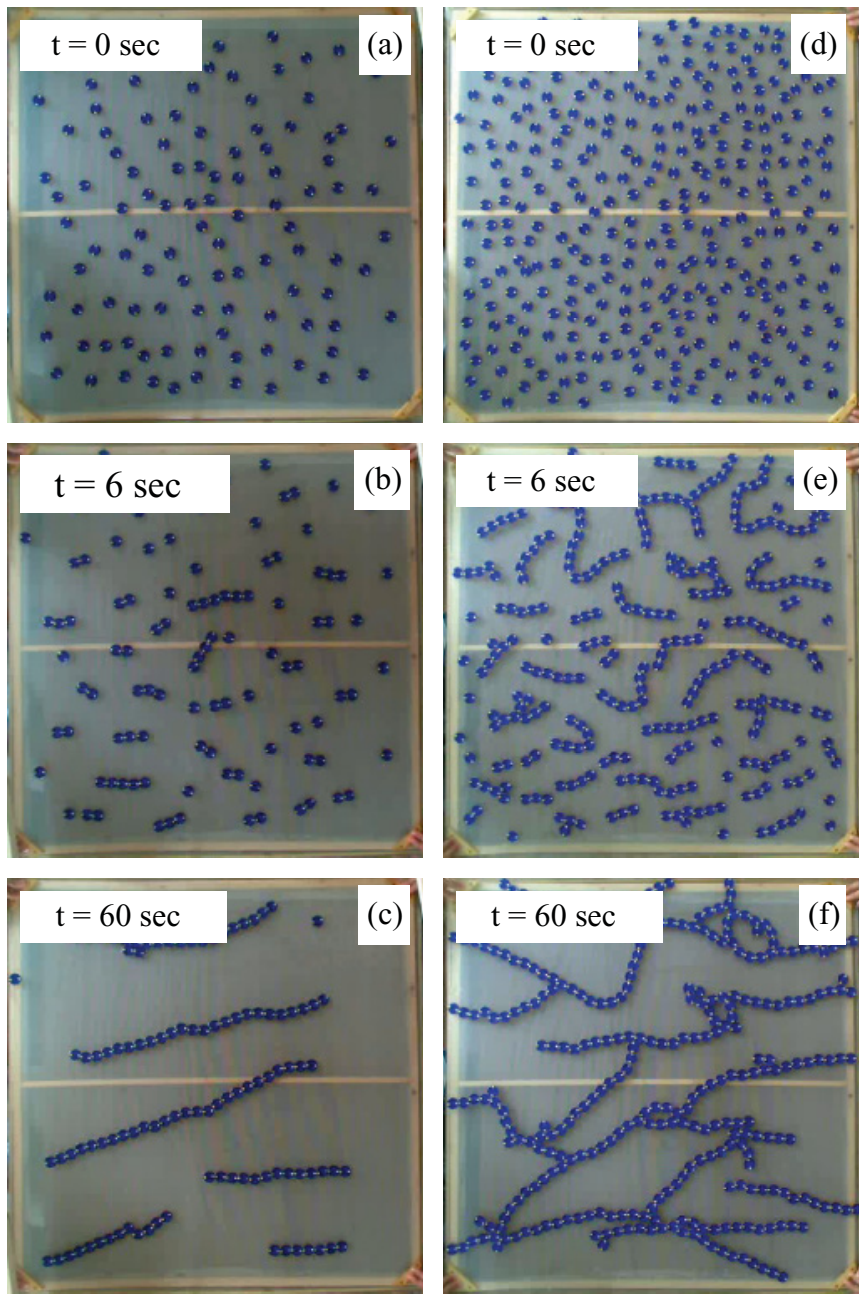

FIG. 2. (Color online) Time evolution of the particle system at two different concentrations $\phi=0.0265$ (a),(b),(c) and $\phi=0.1060$ (d),(e),(f). Cluster-cluster aggregation can be observed where the structure of aggregates strongly depends on the concentration of the system.

limit of our concentration the system rapidly forms a single connected cluster. During this rapid aggregation process it might also occur that more than two clusters merge nearly at the same time, which could only be resolved with a high-speed camera.

Examples of the time evolution of the particle system can be seen in Fig. 2 for two different concentrations $\phi=0.0265$ and $\phi=0.1060$. It can be observed that the system evolves through cluster-cluster aggregation; that is, particles first aggregate and form dimers then trimers which subsequently merge into larger and larger clusters. The system always converges to a nearly frozen state where the time scale of changes becomes very large. The figures also illustrate that the structure of the aggregates strongly depends on the concentration: At low concentration [Figs. 2(a)-2(c)] initially the particles are far enough from each other so that the external magnetic field of Earth dominates the system. It has the consequence that the particles first align with the external field and then aggregate into parallel chains. However, increasing the concentration $\phi$ the local dipole field can overcome the external field, resulting 
in bent chains and branched aggregates which then merge into a single connected cluster with a complex topology [see Figs. 2(d)-2(f)]. We developed a computer code which analyzes all snapshots of the videos, determining the position of the center of particles and also the direction of the vector of dipole moments. Based on the particle positions, clusters can easily be identified as connected sets of touching particles. The size of clusters $S$ is defined as the number of particles involved. It is a very important feature of our system that the particles undergo deterministic motion due to the dipole-dipole interaction. The large particle size ensures that thermal noise cannot have any role in the process. The experimental technique lets us investigate the interaction driven cluster-cluster aggregation of dipolar particles, where the relative importance of the external field and of the dipole-dipole interaction can be controlled by the concentration. In the following we present quantitative results obtained by the image processing technique.

\section{DYNAMICS OF AGGREGATION}

The dynamics of the aggregation process can be characterized by studying the cluster size distribution $n_{S}(t)$ which provides the average number of clusters of size $S$ at time $t$. In a given sample the average cluster size $\langle S\rangle$ and the average number of clusters $\langle n\rangle$ as a function of time can be obtained from $n_{S}(t)$ as

$$
\langle S\rangle(t)=\frac{\sum_{S} n_{S}(t) S^{2}}{\sum_{S} n_{S}(t) S} \quad \text { and } \quad\langle n\rangle(t)=\sum_{S} n_{S}(t),
$$

which are then averaged over samples of different random initial conditions. The results of the data evaluation are presented in Figs. 3 and 4 for the average size and number of clusters, respectively. Note the smoothness of the experimental curves which is due to the high resolution data evaluation and to averaging over a large number of samples. It can be observed in Fig. 3 that $\langle S\rangle(t)$ monotonically increases at

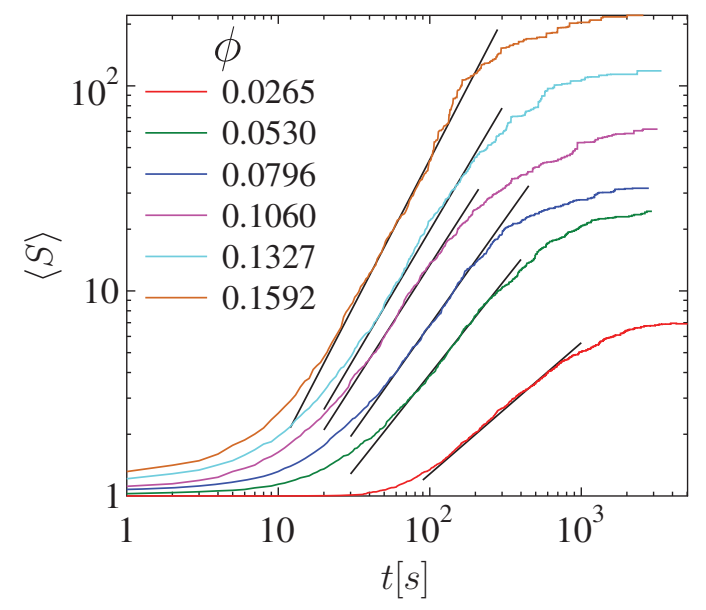

FIG. 3. (Color online) Average cluster size $\langle S\rangle$ as a function of time $t$ for all the concentrations considered (the value of $\phi$ increases from bottom to top at small values of $t$ ). At the time of saturation the aggregation process slows down and the configuration gets practically frozen. For intermediate times the functional form of $\langle S\rangle$ can be well approximated by power laws which are indicated by the straight lines.

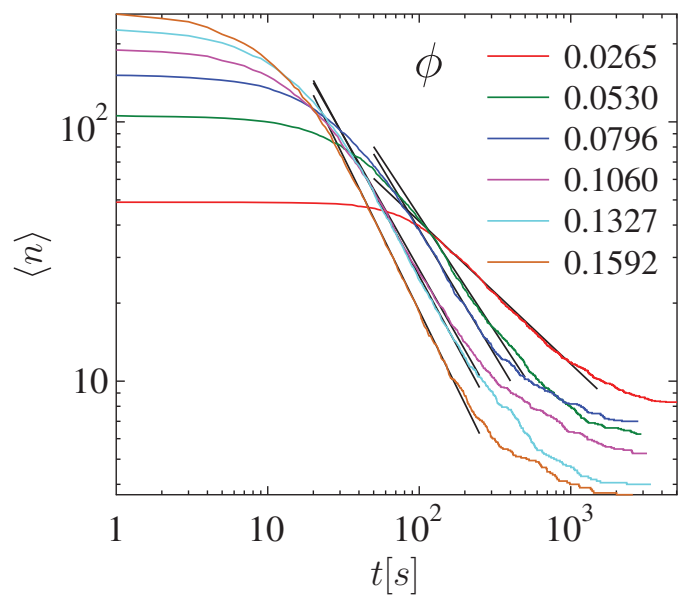

FIG. 4. (Color online) Average number of clusters $\langle n\rangle$ as a function of time $t$ for all the concentrations considered (the value of $\phi$ increases from bottom to top at small values of $t$ ). At intermediate times good quality power law fits can be obtained.

all concentrations and saturates for large times due to the finite size of the system. The average number of clusters $\langle n\rangle$ in Fig. 4 monotonically decreases with time and exhibits the same saturation as $\langle S\rangle$ for large $t$. At low concentration when saturation occurs the growing clusters become so distant that no significant movement can be observed over long durations [see Figs. 2(a)-2(c)]. However, at high concentration saturation is the consequence of the formation of a single connected cluster which gets nearly frozen [see Figs. 2(d)2(f)]. The absence of thermal noise enables the particle system to get trapped in these metastable configurations. It can be seen that for intermediate times both $\langle S\rangle(t)$ and $\langle n\rangle(t)$ can be well approximated by straight lines; that is, they exhibit power law behavior,

$$
\langle S\rangle(t) \sim t^{Z}, \quad\langle n\rangle(t) \sim t^{-Z^{\prime}},
$$

where the dynamic exponents $Z$ and $Z^{\prime}$ are important characteristic quantities of the system. It is also evident in Figs. 3 and 4 that both exponents depend on the concentration such that they both increase with increasing $\phi$. The numerical values of $Z$ and $Z^{\prime}$ obtained by fitting are summarized in Fig. 5 as a function of the concentration.

For the cluster size distribution $n_{S}(t)$ the Vicsek-Family scaling theory of kinetic aggregation predicts the functional form

$$
n_{S}(t) \sim t^{-w} S^{-\tau} f\left(\frac{S}{S^{*}(t)}\right),
$$

where $S^{*}(t)$ is the typical cluster size and $f$ is a cutoff function [26,27]. According to the theory, if $\tau<1$ then $\langle S\rangle(t) \sim S^{*}(t)$ and $\langle n\rangle(t) \sim\left[S^{*}(t)\right]^{-1}$ hold, and, hence, the equality $Z=Z^{\prime}$ follows. The scaling form Eq. (4) provides a good description for diffusion limited cluster-cluster aggregation phenomena [27]. Recently, the scaling relation Eq. (4) has also been found to be valid for magnetic holes aggregating in an external field, when the hole size was sufficiently small [11] making thermal motion possible. In our case $\tau<1$ can be verified and, as expected, in the low concentration regime of Fig. 5 the exponents $Z$ and $Z^{\prime}$ agree very well. Larger deviations are 


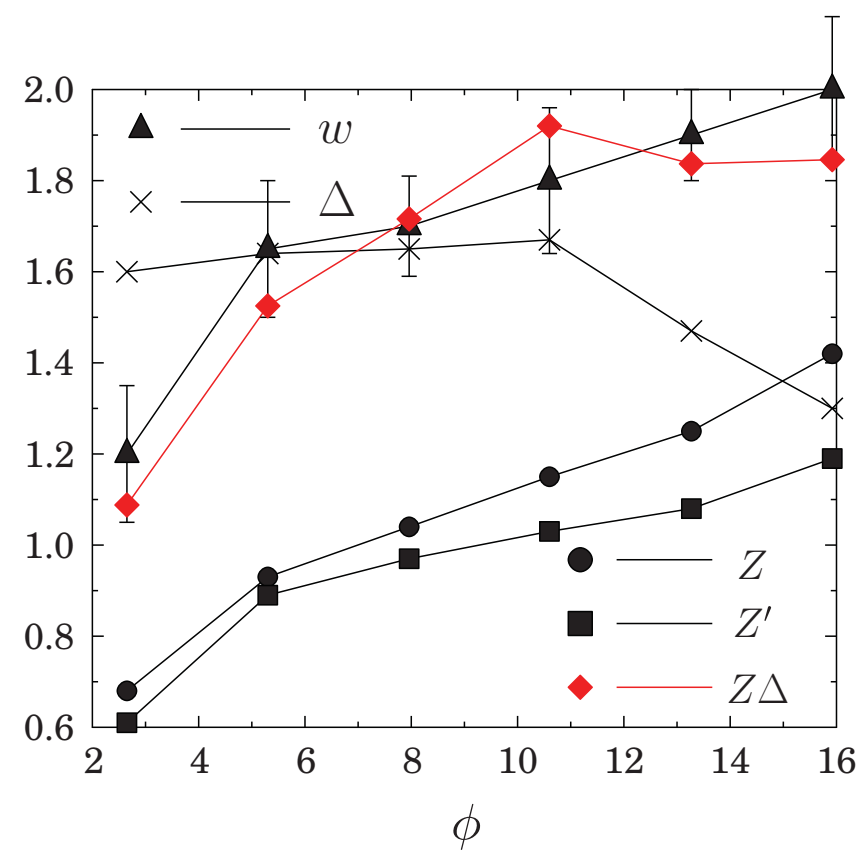

FIG. 5. (Color online) Dynamic exponents characterizing the attraction driven aggregation of dipolar particles as a function of concentration $\phi$. For clarity, error bars are only shown for the exponent $w$. For comparison, we also show the product of the exponents $Z \Delta$ which should be compared to the value of $w$. It can be observed that the exponents fulfill the scaling relation Eq. (6).

only observed for higher concentrations. We emphasize that in our system the motion of particles is fully deterministic; thermal noise or diffusive motion cannot play any role. The experimental data show that the equality of $Z$ and $Z^{\prime}$ remains valid for attraction driven aggregation of in-plane dipoles as well.

It follows from the scaling relation Eq. (4) that for a fixed cluster size $S$ the number or concentration of clusters should asymptotically decay as a power law $n_{S}(t) \sim t^{-w}$ with the exponent $w[26,27]$. The very good statistics of our experimental data enables us to determine the value of $w$ with a reasonable accuracy. Figure 6 presents $n_{S}(t)$ for $S$ values up to 8 as a function of time for four different concentrations. As a reference we always plot the average value of the total number of clusters $\langle n\rangle$ as well. As expected, $n_{S=1}(t)$ decreases monotonically; however, for all cluster sizes larger than $S=1$ the $n_{S}(t)$ curves have a maximum since these clusters are created by the merging of smaller ones. The decreasing part of the curves emerges due to the disappearance of clusters as they join other ones giving rise to larger clusters. In the decreasing regime in all cases the curves can be well approximated by power laws from which we numerically obtained $w$. The values of the exponent $w$ for the different concentrations are presented in Fig. 5, where a weak dependence on the concentration $\phi$ can be observed again.

The cluster size distribution $n_{S}(t)$ can also be written in an alternative form,

$$
n_{S}(t) \sim S^{-2} g\left(S / t^{Z}\right),
$$

where the scaling function $g(x)$ has the property $g(x) \ll 1$ for $x \gg 1$ and it has a power law form $g(x) \sim x^{\Delta}$ for $x \ll 1$
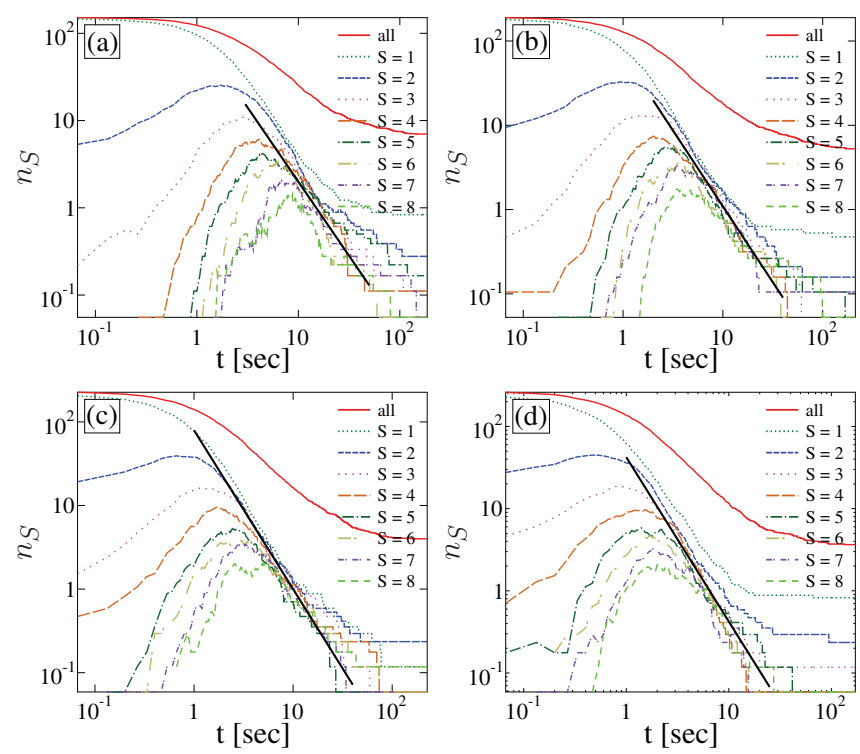

FIG. 6. (Color online) Time evolution of the number of clusters of a fixed size $n_{S}(t)$ for four different concentrations: (a) $\phi=0.0796$, (b) $\phi=0.1060$, (c) $\phi=0.1327$, (d) $\phi=0.1592$. Power laws can be fitted with a reasonable accuracy.

[26,27]. The exponent $\Delta$ of the scaling function is called a crossover exponent. From mass conservation during the aggregation process a relation of the scaling exponents can be derived:

$$
w=Z \Delta .
$$

In order to test the validity of the scaling structure, Eqs. (5) and (6), in Fig. 7 we plot the cluster size distribution $n_{S}(t)$ multiplied by $S^{2}$ as a function of the rescaled cluster size $S /\langle S\rangle$ for several time ranges. To improve the statistics the
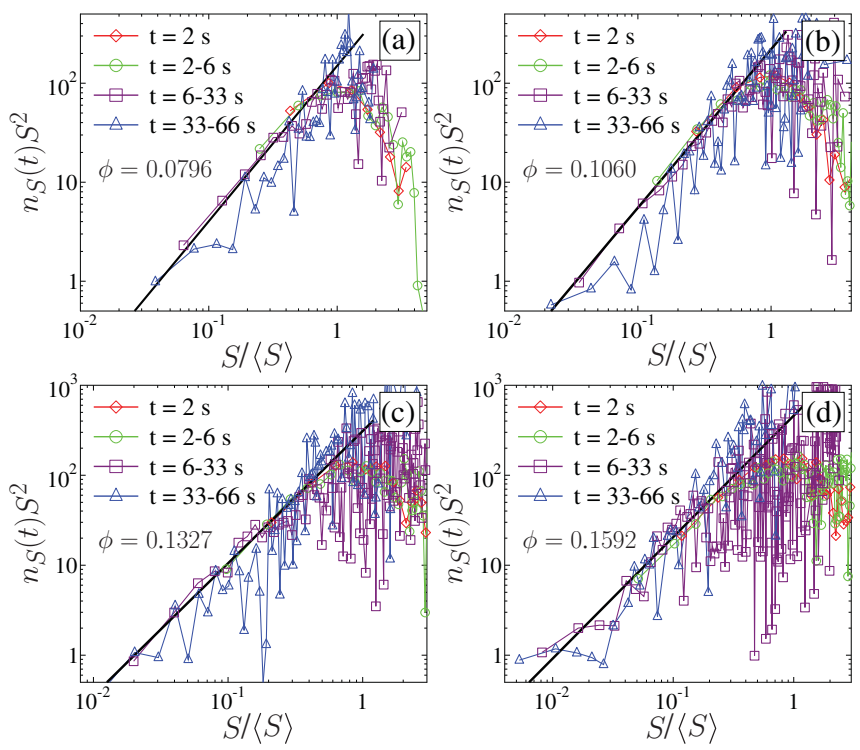

FIG. 7. (Color online) Data collapse analysis of the cluster size distributions obtained at different time intervals. A reasonable collapse of the data is obtained. The value of the crossover exponent $\Delta$ was determined by fitting straight lines. 
$n_{S}(t)$ curves are not presented for single time values but they are averaged over short time intervals. Note that the original $n_{S}(t)$ curves are quite smooth, the larger fluctuations in Fig. 7 arise due to the multiplication by $S^{2}$. The good quality collapse of $n_{S}(t)$ obtained at different times supports the validity of the functional form Eq. (5). We emphasize that in the parameter range $S /\langle S\rangle<1$ the data can be well described by a power law for those time values which belong to the power law regime of $\langle S\rangle$ in Fig. 3. Fitting straight lines in Fig. 7 we determined the value of the exponent $\Delta$, which is again presented in Fig. 5 as a function of $\phi$. It can be observed in Fig. 5 that $Z, Z^{\prime}$, and $w$ are all monotonically increasing with $\phi$; however, for the two highest concentration values the crossover exponent $\Delta$ slightly decreases. Note that all values of the crossover exponent $\Delta$ are larger than 1 , which implies the relation $\tau=2-\Delta<1$. This is consistent with the behavior of the average cluster number $\langle n\rangle \sim t^{-Z^{\prime}}$. Using the numerical values of the exponents $w$, $\Delta$, and $Z$ in Fig. 5 we can verify that they fulfill the scaling relation Eq. (6) with a precision of $0.07-0.15$ depending on the concentration.

\section{DISCUSSION}

We presented an experimental study of the aggregation of millimeter sized particles having a permanent magnetic moment in a weak homogeneous external magnetic field. Due to the large particle size, Brownian motion does not play any role in the system, the particles undergo deterministic motion driven by the mutual attraction. The particles are magnetized to saturation so that the homogeneous magnetic field of Earth just rotates the particles; motion occurs under the action of the dipole-dipole force. Varying the concentration in a broad range we analyzed the time evolution of cluster-cluster aggregation and presented the results in the framework of dynamic scaling theory. At each concentration, experiments were repeated 30 times with random initial positions and in-plane dipole directions. The well controlled conditions of the experiments and the digital image processing enabled us to obtain good quality results for all the quantities of interest of the aggregation process. That the exponent $w$ here is directly determined from experimental data for magnetic particle systems allowed us test the validity of the scaling relation of dynamic exponents.

The experiments revealed that the interaction driven aggregation of dipolar particles obeys the Vicsek-Family dynamic scaling. The scaling relations of the exponents $Z=Z^{\prime}$ and $w=Z \Delta$ are fulfilled with a reasonable accuracy; deviations increase with increasing concentration. Most of the experimental studies in the literature are focused on systems where the particle size (from nano- to micrometer) enables the diffusive motion of the particles. Transition from diffusive to ballistic regime is usually obtained by increasing the strength of interaction which is controlled by the strength of the external magnetic field. It is interesting to note that the values of the exponents $Z$ and $Z^{\prime}$ at our lowest concentration (see Fig. 5) agree well with the ones of Ref. [11] obtained for the largest particles whose motion is mainly ballistic. The increase of $Z$ and $Z^{\prime}$ with the concentration in our system can be attributed to the change of the mobility and of the interaction of growing clusters as the concentration increases. In Ref. [28] an anisotropic cluster-cluster aggregation was studied by means of computer simulations for several values of the exponent $\gamma$ that relates the mobility $D$ to the size of the clusters $D \sim S^{\gamma}$ (see also Ref. [27]). For $\gamma=-0.5$ for the dynamic exponents $Z=Z^{\prime}=0.63 / 0.64$ and $w=1.22$ were obtained [28], which are very close to our experimental results for the lowest density. For $\gamma=0$ the same simulations yielded $Z=Z^{\prime}=1$ and $w=1.75$, which fall very close to the exponents we obtained experimentally at intermediate densities $(\phi \approx 0.08)$. This comparison demonstrates that at low and intermediate densities the experimental results can be interpreted as an anisotropic aggregation with a dependence of the mobility on cluster size that is varying with concentration. Computer simulations of isotropic cluster-cluster aggregation in Ref. [26] provided exponents $Z=1.4$ and $\Delta=1.25$, which fall close to our measured exponents at high concentrations. Therefore, it seems that when increasing the density the aggregation becomes more isotropic in spite of the anisotropic particle-particle interaction.

Studying aggregation processes of dipolar particles, it would be interesting to consider also the effect of the system size on the dynamic exponents. In our experimental setup it could be achieved by decreasing the size of the composite particles or by increasing the size of the container. By carefully exploring all these possibilities it turned out that at smaller sizes the composite particles cannot remain stable on the water surface: When particles merge they may jump on the top of each other, which would modify the outcome of the process. The size of the container cannot be significantly increased either because we cannot ensure that all the particles start to move at the same time. The present setup proved to provide reliable results; however, no size scaling could be studied.

Recently, it has been shown experimentally that the dynamics of the aggregation process of dipolar particles and the resulting structure has some dependence on the shape of the magnetic field [20]. In our case similar questions could be addressed by varying the height of the magnetized cylinder $h_{c}$ at a fixed size of the cork disk $R$. This way we can tune the shape of the magnetic field of the particles from a pointlike dipole to an extended distribution of magnetic moments. Another interesting aspect of our experimental system is that it allows us to investigate the structural change of the system as the concentration is varied. Increasing the concentration beyond the regime of kinetic aggregation, the rapid reorganization of particles gives rise to connected particle sets with a highly nontrivial topology. Work in these directions is in progress.

\section{ACKNOWLEDGMENTS}

This work is supported by the TAMOP-4.2.1/B09/1/KONV-2010-0007 project and by Chinese-Hungarian Intergovernmental Project No. 27/2007. The project is implemented through the New Hungary Development Plan, cofinanced by the European Social Fund and the European Regional Development Fund. F. Kun acknowledges support of the Bolyai Janos project of the Hungarian Academy of Sciences and of OTKA K84157. 
[1] B. M. Berkovsky, V. F. Medvedev, and M. S. Krakov, Magnetic Fluids-Engineering Applications (Cambridge University Press, Oxford, 1993).

[2] P. D. Duncan and P. J. Camp, Phys. Rev. Lett. 97, 107202 (2006).

[3] J. M. Tavares, J. J. Weis, and M. M. Telo da Gama, Phys. Rev. E 73, 041507 (2006).

[4] M. Klokkenburg, R. P. A. Dullens, W. K. Kegel, B. H. Erné, and A. P. Philipse, Phys. Rev. Lett. 96, 037203 (2006).

[5] P. G. de Gennes and P. A. Pincus, Phys. Kondens. Mater. 11, 189 (1970).

[6] A. Ghazali and J. C. Levy, Phys. Rev. B 67, 064409 (2003).

[7] M. Adrian and L. E. Helseth, Phys. Rev. E 77, 021403 (2008).

[8] E. M. Furst and A. P. Gast, Phys. Rev. E 61, 6732 (2000).

[9] S. L. Biswal and A. P. Gast, Phys. Rev. E 68, 021402 (2003).

[10] M. Klokkenburg, B. H. Erné, J. D. Meeldijk, A. Wiedenmann, A. V. Petukhov, R. P. A. Dullens, and A. P. Philipse, Phys. Rev. Lett. 97, 185702 (2006).

[11] J. Cernak, G. Helgesen, and A. T. Skjeltorp, Phys. Rev. E 70, 031504 (2004).

[12] W. Wen, L. Zhang, and P. Sheng, Phys. Rev. Lett. 85, 5464 (2000).

[13] Y. G. Cao and Q. X. Li, Physica A 387, 4755 (2008).

[14] W. Wen, F. Kun, K. F. Pál, D. W. Zheng, and K. N. Tu, Phys. Rev. E 59, 4758 (1999).
[15] F. Kun, W. Wen, K. F. Pál, and K. N. Tu, Phys. Rev. E 64, 061503 (2001).

[16] G. Helgesen, A. T. Skjeltorp, P. M. Mors, R. Botet, and R. Jullien, Phys. Rev. Lett. 61, 1736 (1988).

[17] E. M. Furst and A. P. Gast, Phys. Rev. Lett. 82, 4130 (1999).

[18] D. L. Blair and A. Kudrolli, Phys. Rev. E 67, 021302 (2003).

[19] J. Stambaugh, D. P. Lathrop, E. Ott, and W. Losert, Phys. Rev. E 68, 026207 (2003).

[20] S. K. Hajra and D. V. Khakhar, Phys. Rev. E 69, 031304 (2004).

[21] D. Lopez and F. Petrelis, Phys. Rev. Lett. 104, 158001 (2010).

[22] S. Fazekas, J. Kertész, and D. E. Wolf, Phys. Rev. E 68, 041102 (2003).

[23] I. Varga, H. Yamada, F. Kun, H. G. Matuttis, and N. Ito, Phys. Rev. E 71, 051405 (2005).

[24] BorsMagnet Co., Budapest, Hungary [http://www.borsmagnet. $\mathrm{hu} / \mathrm{l}$.

[25] I. Varga, N. Yoshioka, F. Kun, S. Gang, and N. Ito, J. Stat. Mech. (2007) P09015.

[26] T. Vicsek and F. Family, Phys. Rev. Lett. 52, 1669 (1984).

[27] T. Vicsek, Fractal Growth Phenomena (World Scientific, Singapore, 1989).

[28] S. Miyazima, P. Meakin, and F. Family, Phys. Rev. A 36, 1421 (1987). 